\title{
Metabolic regulation of CAR T cell function by the hypoxic microenvironment in solid tumors
}

\author{
Anna Schurich*, ${ }^{*}$, Isabelle Magalhaes**, $\$, 2$ \& Jonas Mattsson ${ }^{2,3}$ \\ ${ }^{1}$ School of Immunology and Microbial Sciences, King's College London, London, UK \\ ${ }^{2}$ Department of Oncology-Pathology, Karolinska Institutet, Stockholm, Sweden \\ ${ }^{3}$ Clinical Immunology, Karolinska University Hospital, Huddinge, Sweden \\ *Author for correspondence: anna.schurich@kcl.ac.uk \\ **Author for correspondence: Isabelle.Magalhaes@ki.se \\ ${ }^{\ddagger}$ Authors contributed equally
}

The field of immunometabolism has attracted growing attention as an area at the heart of immune regulation. Upon activation, T cells undergo significant metabolic changes allowing them to mediate effector responses. The advent of chimeric antigen receptor T cell-adoptive therapy has shown some striking clinical efficacy but fails to induce sufficient antitumor response in many patients. Solid tumors put up significant opposition creating a microenvironment deficient of oxygen and glucose, depriving $T$ cells of energy and pushing them to exhaustion. Here, we focus on immune suppressive mechanisms related to hypoxia in the tumor microenvironment and the resulting metabolic changes in T cells. New therapeutic approaches such as generating chimeric antigen receptor $\mathrm{T}$ cells able to withstand the challenging solid tumor microenvironment are needed.

First draft submitted: 10 September 2017; Accepted for publication: 10 December 2018; Published online: 25 January 2019

Keywords: cancer therapy $\bullet$ CAR T cells $\bullet$ hypoxia $\bullet$ metabolism $\bullet T$ cell engineering $\bullet T$ cell exhaustion $\bullet$ tumor microenvironment

Despite recent unprecedented improvements in cancer therapy due to the rise of immunotherapeutic treatments like the use of immune checkpoint inhibitors (awarded the 2018 Nobel Prize in Physiology or Medicine), many patients still do not respond to treatment and succumb to cancer [1]. In the EU in 2015, cancer caused more than $25 \%$ of all deaths [2].

A better understanding of tumor biology and immunology (in addition to other mechanisms) is direly needed. In this review, we will specifically address the challenges met by cytotoxic $\mathrm{T}$ cells in the tumor microenvironment (TME).

Solid tumor microenvironments are complex tissues composed of malignant but also nonmalignant cells (e.g., stromal and immune cells), extracellular matrix, blood and lymphatic vessels [3].

The tumor microenvironment is known to be immunosuppressive due to a plethora of contributing mechanisms. These include altered expression of chemokines resulting in poor inflammatory cell trafficking to the tumor, recruitment or induction of suppressive cell populations, production of immune suppressive cytokines, expression of co-inhibitory ligands and depletion of vital nutrients [4-6]. Oxygen is an essential part of cellular energy metabolism. Low $\mathrm{O}_{2}$ levels (hypoxia) are often found in the solid tumor microenvironment and are attributed (among other factors) to the rapid growth of tumor cells and inadequate blood flow due to heterogeneous perfusion [7]. The atmospheric value of $\mathrm{O}_{2}$ (normoxia) is around $21 \%$, while in tissues (physioxia) the fraction of $\mathrm{O}_{2}$ can range from $1 \%$ (skin) to $14 \%$ (lung) [8]. For tumors, it has been reported to be for example $2.2 \%$ in non-small-cell lung cancer [9] and as low as $0.3 \%$ in pancreatic cancer [10].

These suppressive influences are employed in various combinations by different tumors, creating significant challenges for the immune response and immune-based therapy. Those mechanisms impacting the metabolic operation of immune cells are only starting to be unraveled. 


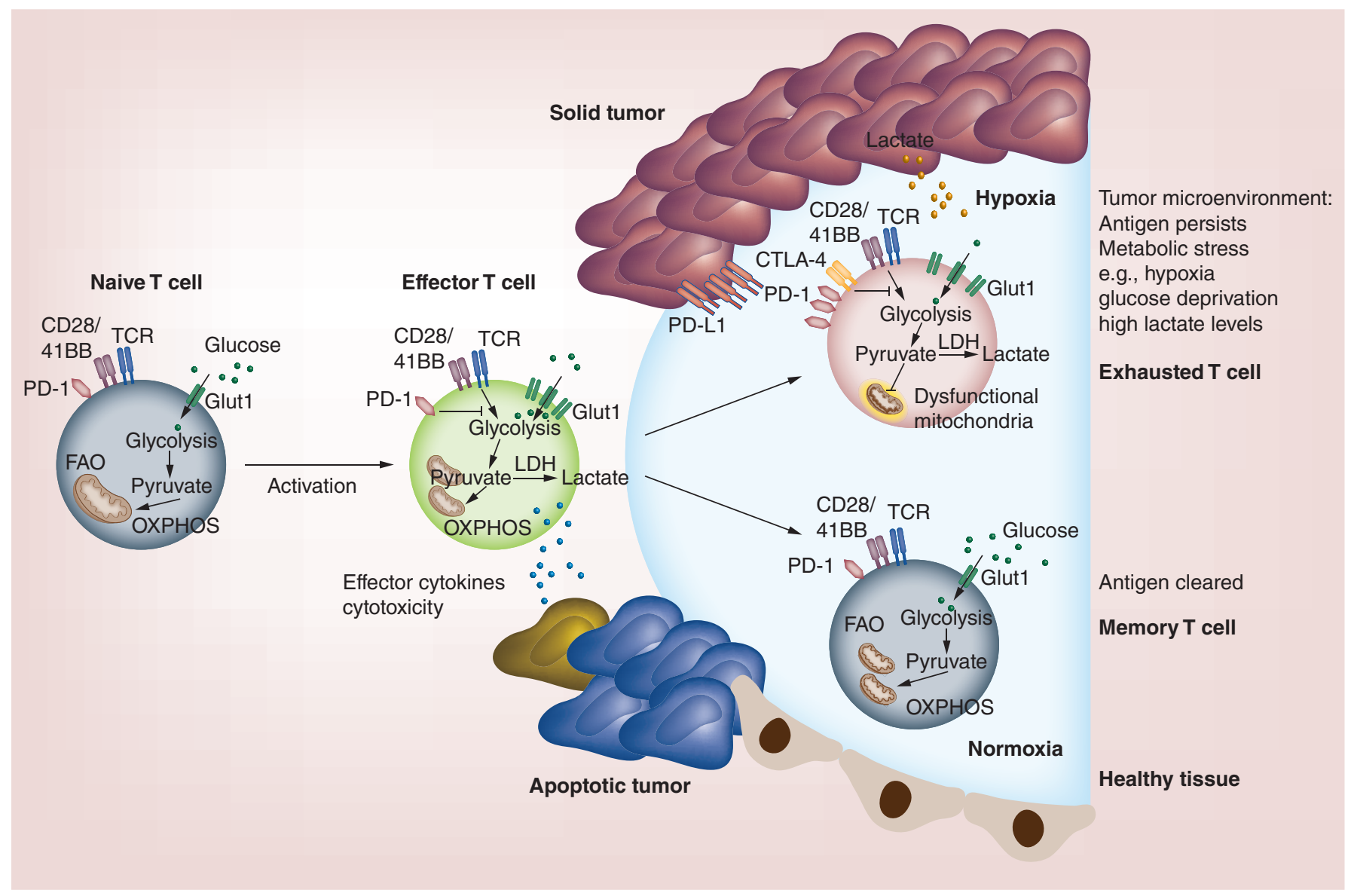

Figure 1. T cell activation and metabolism. Left hand side: Naive T cells mainly use oxidative phosphorylation (OXPHOS) and fatty acid metabolism. Middle: Upon activation glucose transporter-1 (Glut-1) is upregulated and glycolysis is increased in effector cells. To maintain a high glycolytic rate pyruvate is converted by the enzyme lactate dehydrogenase into lactate. Glycolysis allows T cells to rapidly divide and produce effector cytokines and cytotoxic mediators. Lower right hand side: When antigen, such as abnormal cells, can be cleared the T cell response contracts and memory T cells develop. Memory T cells revert back to a metabolism similar of that of naive cells, but remodel their mitochondria allowing them to rapidly produce energy for a recall response upon antigen re-encouter. Upper right hand side: However, when antigen cannot be cleared and persists, for example when abnormal cells overwhelm the immune response and establish a progressive solid tumor T cells become exhausted. Solid tumors often express co-inhibitory molecules such as PD-L1 dampening down $T$ cell responses and the hypoxic, high lactacte, low glucose microenvironment further suppresses immune cells. Exhausted T cells albeit upregulating Glut-1 cannot take up sufficient energy in the nutrient-depleted tumor microenvironment. These T cells show mitochondrial dysfunction, express high levels of co-inhibitory receptors and show low capacity for proliferation and effector function. PD-L1: Programmed death-ligand 1.

Sharping et al. recently noted that: [the] "metabolism represents a key mechanism through which immune cells can be regulated" [11]. This statement seems especially true in the context of cancer and we will discuss the effect of metabolic stress (with a focus on hypoxia) imposed on T cells in the TME below.

\section{Metabolic regulation of T cells}

$\mathrm{T}$ cells provide effective protection against intracellular pathogens and usually eliminate abnormal cells before they develop into cancer. Although $\mathrm{CD}^{+} \mathrm{T}$ cells orchestrate adaptive immune responses, $\mathrm{CD} 8^{+} \mathrm{T}$ cells constitute the main effector arm. To meet the immense energetic demands of rapid proliferation, differentiation, mobilization and production of effector molecules, T cells need to modulate their metabolism accordingly.

During activation, $T$ cells switch from the mainly oxidative and fatty acid metabolism of naive and resting $\mathrm{T}$ cells to increased glucose uptake and glycolysis (Figure 1) [12]. In glycolysis, glucose is broken down into pyruvate resulting in a net gain of two ATP molecules and the conversion of two co-enzymes NAD+ to NADH. Pyruvate can then be shuttled into the tricarboxylic acid cycle fuelling oxidative phosphorylation (OXPHOS). Glycolysis, albeit yielding little energy per glucose molecule metabolized, allows for rapid production of substrates for the 
biosynthesis of amino acids, lipids and nucleotides, which are essential for actively dividing cells. To maintain a high glycolytic rate, NADH needs to be converted back into NAD+ and this is achieved by reducing pyruvate to lactate, a reaction catalyzed by the enzyme lactate dehydrogenase. Glycolysis, which can run in anaerobic conditions, is favored by rapidly dividing cells even in the presence of sufficient oxygen, a process termed aerobic glycolysis and first observed by Warburg in tumor cells [13].

However, during initial $\mathrm{T}$ cell activation, $\mathrm{T}$ cells cluster together likely creating an area of low oxygen tension, which might further promote glycolysis [8]. Inhibition of this key metabolic switch to glycolysis inhibits full $\mathrm{T}$ cell activation [12]. In support, tumor-infiltrating lymphocytes (TILs) purified from low glucose tumor environment as well as $\mathrm{T}$ cells experimentally cultured under limiting glucose conditions showed a 'glucose deprivation signature' and were functionally impaired [14].

Once the antigen is cleared (in case of an acute insult) the $\mathrm{T}$ cell response contracts and memory $\mathrm{T}$ cells are formed. A requirement for $\mathrm{T}$ cell memory induction is to revert back to primarily oxidative and fatty acid metabolism and $\mathrm{T}$ cells remodel their mitochondria in this process $[4,15]$.

Thus, $\mathrm{T}$ cell differentiation status and metabolic profile are intimately linked, with broadly naive and memory $\mathrm{T}$ cells preferentially using OXPHOS, effector $\mathrm{T}$ cells using increased glycolysis and regulatory $\mathrm{T}$ cells (Treg) favoring fatty acid oxidation [4]. Less well-characterized subsets, such as resident $\mathrm{T}$ cells [16], terminally differentiated/senescent $T$ cells [17] and exhausted $T$ cells come with their own demands.

To add to the complexity, $\mathrm{CD}^{+}$and $\mathrm{CD}^{+} \mathrm{T}$ cells have different metabolic requirements [18]. For example, $\mathrm{CD}^{+} \mathrm{T}$ cells have been described to make better use of glutamine/glutaminolysis in the absence of glucose than $\mathrm{CD}^{+} \mathrm{T}$ cells. This higher glycolytic metabolism allowed $\mathrm{CD}^{+} \mathrm{T}$ cells to divide more rapidly upon activation and made them less sensitive to metabolic inhibition under the experimental settings. In comparison, $\mathrm{CD}^{+}{ }^{+} \mathrm{T}$ cells were relatively more dependent on OXPHOS $[18]$. These findings might indicate that $\mathrm{CD} 4^{+} \mathrm{T}$ cells are less capable of functioning in hypoxic environments, such as those found in the tumor microenvironment.

Despite the importance of increased glycolysis, effector $T$ cells must maintain mitochondrial oxidative metabolism for optimal effector function [12,15]. Indeed, disruption of mitochondrial function seems to be a major contributing factor to the ailing $\mathrm{T}$ cell response called exhaustion $[11,19,20]$, which will be discussed below. In support of a positive impact of mitochondria, Kawalekar et al. found that using chimeric antigen receptor (CAR) T cell constructs with a co-stimulatory 4-1BB signaling domain stimulated mitochondrial biogenesis and OXPHOS resulting in prolonged in vitro persistence [21].

In summary, the cellular metabolic profile is shaped by signaling via the T cell receptor (TCR) together with co-stimulation or co-inhibition as well as metabolite availability in the environment. These complex processes have yet to be better understood in order to facilitate successful modulation of $\mathrm{T}$ cell responses for therapy. Different settings might call for $\mathrm{CD}^{+}$instead of $\mathrm{CD}^{+} \mathrm{T}$ cells or vice versa, using varying proportions of memory versus effector cells, optimizing signaling domains, in addition to producing therapeutic $\mathrm{T}$ cells under conditions that prime a metabolic profile that enables them to withstand the immune suppressive tumor microenvironment.

\section{T cell exhaustion in the tumor microenvironment}

In a situation of persistent antigenic stimulation or ongoing inflammation, $T$ cells do not develop into memory cells but undergo phenotypic, functional and metabolic changes, which result in $\mathrm{T}$ cell exhaustion (Figure 1). It remains to be elucidated which is cause and which is effect. T cell exhaustion was first observed in chronic viral infections but is now recognized to also occur in cancer [22]. In a recent review, Zhang and Ertl raised an interesting question: is $T$ cell exhaustion caused by metabolic stress, in other words, low oxygen and nutrient availability, rather than overwhelming antigenic stimulation? They argue that in tumors, antigens might only be expressed at low level and presentation can be limited by MHC class I downregulation [6], therefore ongoing antigenic stimulation might not be the dominant driver of $\mathrm{T}$ cell dysfunction in the tumor microenvironment.

Exhaustion is characterized by a hierarchical loss of effector functions [23]. T cell endogenous IL-2 production wanes first leading to reduced proliferation. This is followed by a loss of cytotoxicity and then reduction and finally loss of effector cytokine production, with IFN- $\gamma$ being lost last. Severely exhausted T cells become prone to apoptosis and are deleted from the $T$ cell pool [24]. Exhausted $T$ cells can express multiple co-inhibitory receptors, such as programmed death-1 (PD-1), cytotoxic T lymphocyte antigen-4 (CTLA-4), lymphocyte activation gene-3, $\mathrm{T}$ cell immunoglobulin and mucin domain 3 (Tim-3) and others in various combinations [22]. PD-1 is often described as the hallmark receptor of $\mathrm{T}$ cell exhaustion [22]. Signaling through these upregulated co-inhibitory receptors suppresses $\mathrm{T}$ cell activation and dampens-down ongoing immune responses. For example, PD- 1 and CTLA- 4 
interfere, through distinct mechanisms, with co-stimulatory signaling through CD28 [25] with downstream effects on the cellular metabolism [26].

\section{Suppressive mechanisms in the tumor microenvironment}

The majority of cancer-related mortality and morbidity originates from solid tumors which develop and metastasize [5]. Due to their commonly chaotic structure, solid tumors are prone to containing areas of low oxygen tension. Hypoxia can lead to metabolic stress which can in turn suppress the immune response, allowing the tumor to grow and metastasize [27] As an emerging concept, Hanahan et al. argued that metabolic alterations in tumor cells and tumor microenvironment should be added to the existing catalog for the hallmarks of cancer [27].

Hypoxia induces upregulation of the glucose transporter-1 (GLUT-1) and increased glycolysis in tumor cells via induction of hypoxia-inducible factor-1 $\alpha$ (HIF-1 $\alpha$ ) [27]. Increased glycolysis delivers the substrates for the largescale biosynthetic processes of tumor cell growth and rapid cell division [27]. Under hypoxic conditions glucose is fermented to lactate. In the TME lactate can reach immune suppressive concentrations of $20-30 \mathrm{mM}$ as opposed to around $3 \mathrm{mM}$ in healthy tissue [28]. Lactate has been implicated in dampening of $\mathrm{T}$ and NK cell effector functions (cytokine production and cytotoxicity) and survival [29] as well as reducing TNF production by macrophages [30]. An inverse correlation between the glycolytic activity of tumor cells and $\mathrm{T}$ cell killing has been demonstrated and blocking tumor lactate dehydrogenase significantly increased tumor susceptibility to $T$ cell killing [31]. One mechanism allowing enhanced killing might be the inhibition of lactate-stimulated upregulation of PD-L1 on tumor cells, as has been demonstrated in lung cancer cells [32].

In $\mathrm{T}$ cells, hypoxia decreased the expression of the $\mathrm{T}$ effector cell transcription factor T-bet leading to an increased expression of co-inhibitory receptors CTLA-4, lymphocyte activation gene-3 and 2B4 [33] and hence less active T cells. Indeed, overexpressing T-bet in CD4 CAR T cells in a murine model increased their tumor efficacy [34]

A recent study showed that $\mathrm{T}$ cells entering the tumor microenvironment can undergo loss of mitochondrial function in part due to defective mitochondrial biogenesis [11]. Mitochondrial depolarization and mitochondrial loss are linked to $\mathrm{T}$ cell exhaustion in cancer and chronic viral infections, leaving effector cells unable to fuel an efficient immune response even when glycolysis was increased $[11,19,20]$.

The hypoxic tumor microenvironment favors a glycolytic over an oxidative metabolism and enhanced glycolysis and increased lactate production in tumors correlates with more aggressive forms of the disease with worse prognosis [35]. Unfortunately, glycolytic tumor cells outcompete $\mathrm{T}$ cells effectively starving them by depleting the tumor microenvironment of glucose [6,36]. In addition, levels of essential amino acids in the tumor microenvironment can be low. Tumor infiltrating cell populations like myeloid-derived suppressor cells produce arginase, depleting arginine, while tumor-associated fibroblasts can break down the amino acid tryptophan, which also yields kynurenine a suppressive metabolite $[4,37]$. Thus, TIL have limited metabolic choices in the tumor microenvironment and this significantly impairs their effectiveness.

\section{Hypoxia in tumors, impact of HIF-1 $\alpha$ on T cells}

Hypoxia has been described in different solid tumors [38] and was already recognized in the 1950's to induce tumor resistance to anticancer therapies such as radiotherapy [39]. The HIF transcription factors play a central role in cell adaptation to hypoxic stress. HIFs are dimers composed of the oxygen-labile HIF- $\alpha$ (the isoforms HIF-1 $\alpha$, HIF- $2 \alpha$ or HIF- $3 \alpha$ ) and the constitutively expressed $\beta$ subunit (usually HIF-1 $\beta$ ). HIF- $1 \alpha$ (ubiquitously expressed) and HIF-2 $\alpha$ (tissue-specific expression) are structurally similar isoforms that have been extensively studied while HIF-3 $\alpha$ is less well characterized [40]. We will focus below on HIF-1 $\alpha$ in T cells during hypoxia.

Under normoxic conditions, HIF-1 $\alpha$ binds to the von Hippel-Lindau protein that targets HIF- $1 \alpha$ for degradation by the proteasome. During hypoxia, HIF-1 $\alpha$ subunit is stabilized, translocates into the nucleus where it interacts with HIF-1 $\beta$ and subsequently binds to hypoxia-responsive elements on the promoters or enhancers of targeted genes [41]. Hypoxia and HIF-1 $\alpha$ influence not only tumor cells but also tumor-infiltrating immune cells. Indeed, HIF-1 $\alpha$ protein is widely expressed and detected in most human tumors [42], as well as in innate and adaptive immune cells [43].

HIF- $1 \alpha$ is an important regulator of cellular metabolism. It induces the expression of glycolytic proteins, driving cells to switch from oxidative phosphorylation toward glycolysis [41].

HIF-1 $\alpha$ impacts the various T cell subsets differently. HIF-1 $\alpha$ has been shown to modulate the balance between Treg and Th17 cells in murine models, with some reports demonstrating that HIF- $1 \alpha$ inhibited Tregs and promoted Th17 cells $[44,45]$. However, in other studies in human cells, HIF-1 $\alpha$ mediated the inhibition of Treg 
differentiation [46], while others have shown HIF-1 $\alpha$ promoted FoxP3 [47,48] under hypoxic conditions. Therefore, the role of HIF- $1 \alpha$ in Tregs remains to be understood.

The impact of HIF-1 $\alpha$ on T cells in vivo remains controversial. In a murine sepsis model, animals with HIF$1 \alpha$-deficient T cells showed increased survival and displayed increased levels of TNF and IL- 6 in peritoneal lavage fluid and blood serum compared with wild-type animals [49]. This suggested that HIF-1 $\alpha$ inhibits T cell activation in the hypoxic inflamed peritoneum. Zhang et al. showed that HIF-1 $\alpha$ deficient CD8 ${ }^{+}$OT-1 tumor infiltrating $\mathrm{T}$ cells were more polyfunctional. These cells showed reduced glycolysis but enhanced fatty-acid catabolism, and B16 Ova tumor progression was delayed in mice that received HIF-1 $\alpha$ deficient CD8 ${ }^{+}$OT-1 T cells [50]. In contrast, other reports indicate that HIF- $1 \alpha$ may enhance $\mathrm{CD}^{+} \mathrm{T}$ cell functions. Mice with von Hippel-Lindau-deficient OT-1 CD $8^{+}$T cells (with enhanced HIF- $1 \alpha$ and HIF-2 $\alpha$ activity) showed increased control of B16 OVA tumor growth [33]. In accordance with this finding, more recently Palazon et al. reported that mice with HIF-1 $\alpha$ deficient $\mathrm{T}$ cells, grafted with Lewis lung carcinoma and B16-F10 melanoma HIF-1 $\alpha$, displayed less tumor infiltrating CD8 ${ }^{+}$ $\mathrm{T}$ cells and more rapid tumor growth. Furthermore, HIF-1 $\alpha$-deficient OT-1 T cells had reduced tumor infiltration and capacity to control B16 OvA tumor growth [51]. An interesting in vitro study using human CAR T cells showed that during hypoxia effector memory versus central memory or naive $\mathrm{T}$ cells displayed different expansion capacity, possibly linked to different levels of HIF-1 $\alpha$. Effector memory T cells were enriched in HIF-1 $\alpha$ compared with other subsets, even in the absence of stimulation [52].

Overall, these controversial findings demonstrate that more studies are needed to elucidate the impact of HIF$1 \alpha$ on the effector functions of $\mathrm{CD}^{+}$and $\mathrm{CD}^{+} \mathrm{T}$ cell subsets in the hypoxic tumor environment. This is of particular importance in the context of human $\mathrm{T}$ cell therapies. Furthermore, it is necessary to keep in mind that while conducting in vitro experiments, normoxic conditions usually used as a control for hypoxic studies may not be the best comparator since $\mathrm{O}_{2}$ physiological levels encountered by $\mathrm{T}$ cells (even before infiltrating tumors) are below $\mathrm{O}_{2}$ atmospheric values.

Of note, HIF-1 $\alpha$ in T cells is not only stabilized during hypoxia but also following TCR and cytokine activation $[43]$.

\section{TILs \& CAR T cells in solid tumors}

$\mathrm{T}$ cell-based adoptive cell transfer to treat tumors relies on the infusion of tumor-reactive $\mathrm{T}$ cells. This requires the in vitro expansion of sufficient numbers of tumor-reactive $\mathrm{T}$ cells (to be infused to patients) that are derived either from tumor-reactive $\mathrm{T}$ cells (obtained from patients' tissues) or from genetically modified $\mathrm{T}$ cells that express specific TCRs or CARs directed against tumor antigens.

TILs are a broadly heterogeneous cell population among which tumor-reactive $\mathrm{CD} 8^{+} \mathrm{T}$ cells can be identified. Single cell analysis like single cell RNA sequencing or mass cytometry have allowed the characterization of TILs and show that expression of certain markers, for instance CD39 [53], may allow specific discrimination of tumorreactive $\mathrm{CD} 8^{+}$TILs from bystander CD8 ${ }^{+}$TILs (e.g., reactive against viral antigens like EBV or CMV) [54]. The specific identification of tumor-reactive $\mathrm{CD}^{+} \mathrm{T}$ cells is of utmost importance to allow better characterization (e.g., expression levels of immune checkpoints). This will enable the design of targeted treatments to enhance $\mathrm{T}$ cell effector functions, or help the in vitro production of tumor-reactive $\mathrm{T}$ cells for adoptive cell transfer.

Adoptive cell transfer using in vitro expanded TILs for the treatment of metastatic melanoma was first reported by Rosenberg [55]. To date, various trials using TILs for the treatment of melanoma as well as other solid tumors have been, and are being, conducted. The latest Trial Watch for cancer immunotherapy provides an overview of current trials [56]. TIL therapy for the treatment of metastatic melanoma is the most effective with durable, complete response rates $>20 \%[57]$.

The immunotherapy field was recently boosted with the emergence of CAR T cell therapy [58]. Since their antigen-recognizing receptor is based on modified antibodies, CARs can specifically target surface antigens, such as the CD19 co-receptor on B cells. CAR T cells circumvent the necessity for HLA-class matching [5] opening up the possibility of using allo $\mathrm{T}$ cells in the future.

The use of CD19-directed CAR T cells for the treatment of B-cell malignancies has demonstrated impressive response rates [59] (as high as $83-93 \%$ in adults with B cell acute leukemia). However, the potency of CAR T cells comes at a price, with some patients developing severe side effects: cytokine release syndrome (associated with tumor burden) and/or neurotoxicity. Many efforts are now made to apply CAR T cells for the treatment of other hematological cancers and solid tumors such as lung and pancreatic cancers [60]. Some results from clinical trials targeting solid tumors have been reported. The results are not nearly as impressive [61] as for CD19 CAR 


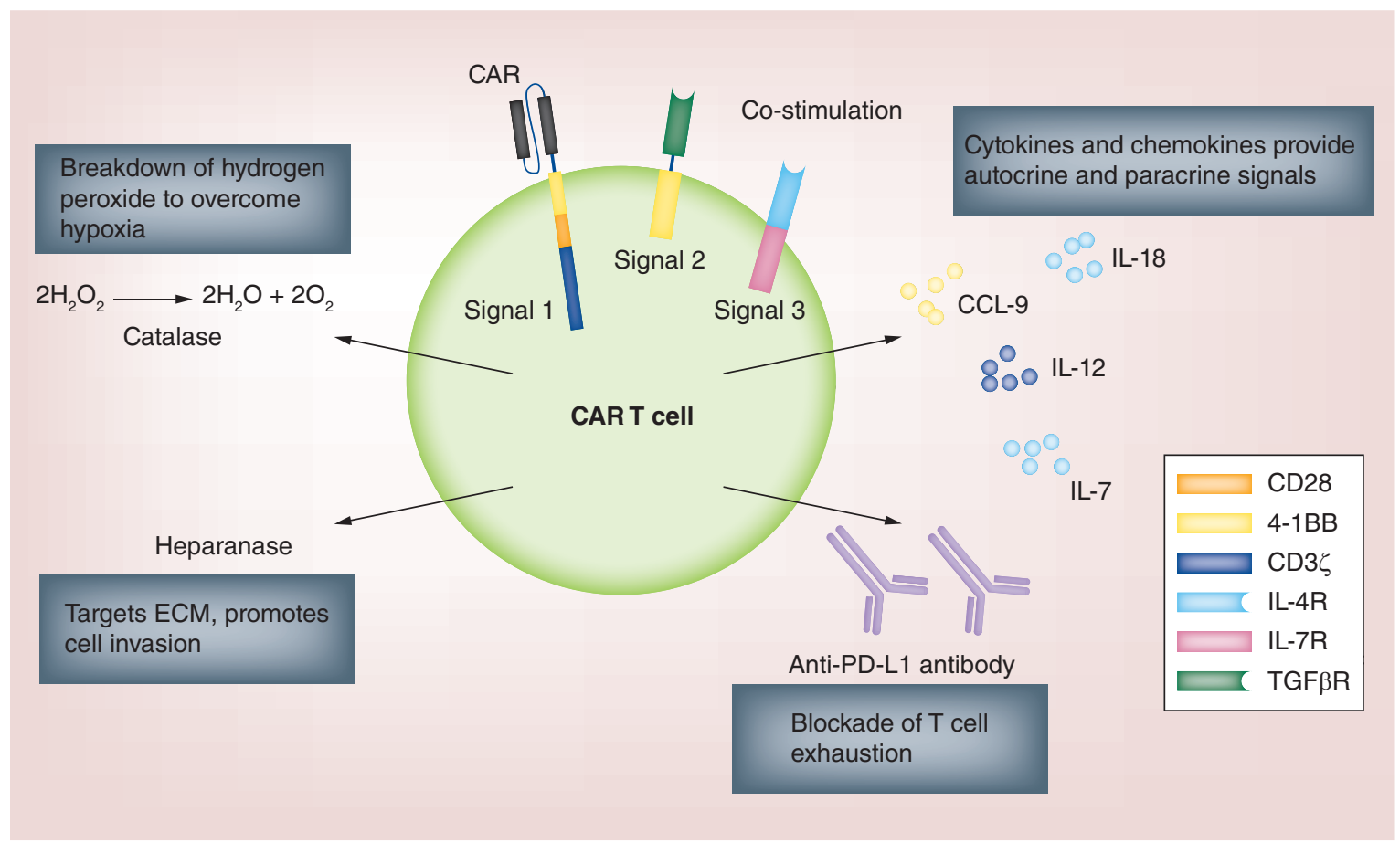

Figure 2. Armored CAR T cells counteract the tumor microenvironment. Middle panel: $T$ cells expressing a CAR construct containing one or two co-signaling domains (e.g., CD28 and 4-1BB that provide signal 2) can be further genetically engineered to improve their functions by co-expressing additional molecules. Upper right hand side panel: CAR T cells can express chimeric cytokine receptors where binding of anti-inflammatory cytokines present in the tumor microenvironment (e.g., TGF- $\beta$, IL-4) deliver a stimulatory via a signal 2 or signal 3 via the fused signalling domain. Middle right hand side panel: Furthermore, CAR T cells secreting cytokines or chemokines can provide signals that not only act in an autocrine but also in a paracrine fashion. Left hand side panel: Additionally, CAR T cells can be modified to secrete enzymes, e.g., catalase, heparanase, or as shown in the bottom right hand side panel, anti-PD-L1 antibodies in order to modify the inhibitory tumor microenvironment and block T cell exhaustion, respectively. CAR: Chimeric antigen receptor; ECM: Extracellular matrix; PD-L1: Programmed death-ligand 1.

T cell treatments, with limited, but promising, antitumor efficacy. Unfortunately, in a few cases fatal toxicity (on target/off-tumor killing of normal epithelial cells) has been reported [62].

The development of new CAR T cell therapies means new challenges: tumor heterogeneity, identification of tumor-specific or tumor-associated antigens that do not mediate serious on-target/off-tumor toxicity, cell dosage, mode of cell delivery (systemic or local), choice of conditioning regimen and because of the potential immunosupressive tumor microenvironment, combination with other immunotherapy, in other words, checkpoint inhibitors. In a case report of a patient with diffuse large B-cell lymphoma, the administration of pembrolizumab (anti-PD-1) after CD19 CAR T cell infusion suggested that pembrolizumab may increase CAR T cell efficacy [63]. However, in patients with neuroblastoma who received antidisialoganglioside CAR T cells, the administration of pembrolizumab (before and after CAR T cell infusion) did not appear to impact CAR T cell efficacy [64].

\section{Modulation of tumor environment by CAR T cells}

Optimal $\mathrm{T}$ cell activation relies on the integration of three signals: TCR, co-stimulation and pro-inflammatory cytokines [65]. Second- and third-generation CAR designs provide CAR T cells with signal 1 (via the CD3- $\zeta$ ) and signal 2 (via co-stimulatory domains) [66], while signal 3 (via cytokines) depends on the cytokines present in the milieu.

In the vast majority of clinical trials, CAR T cells are generated by genetical engineering using retroviral vectors encoding for the CAR construct [66]. Interestingly, CAR T cells can be further modified by genetic engineering, the CAR T cells are then termed armored or fourth-generation CARs [66,67], to overcome the challenges faced by $\mathrm{T}$ cells in the TME, but also to modulate the TME and counteract the TME immune suppression as illustrated below and in Figure 2. 
In the TME, suppressive cells produce immunosuppressive cytokines such as IL-10 and TGF- $\beta$ [68]. The introduction of activating chimeric receptors binding to IL-4 in CAR T cells has allowed to reverse TME immunosuppression and support CAR T cell activation and proliferation [69]. This strategy can be further refined as illustrated in a recent report, where CAR T cells were engineered with chimeric receptors consisting of the exodomains of TGF- $\beta$ and the IL-4 receptor fused to the endodomains of the 4-1BB and IL-7 receptor providing signal 2 and 3 to CAR $\mathrm{T}$ cells, respectively [70]. Other approaches rely on the secretion of cytokines by armored CAR T cells [67], for example IL-12 [71] or IL-18 [72]. The secretion of CAR T cell-derived IL-7 and the chemokine CCL19 has been shown to induce potent antitumor responses. Those responses were mediated by CAR T cells in coordination with tumor-reactive (non-CAR) T cells via epitope spreading [73]. Additionally, immune exhaustion due to checkpoint blockade can be overcome by using CAR T cells secreting an anti-PD-L1 antibody [74] with the advantage of intratumor delivery as compared with conventional infused antibody treatment. CAR T cells expressing the enzyme catalase protect CAR T cells and bystander cells from oxidative stress in vitro [75], which in the context of the TME could benefit CAR T cells, but also other tumor-infiltrating immune cells. The expression by CAR T cells of the enzyme heparanase that degrades components of the extracellular matrix was shown to promote better tumor $\mathrm{T}$ cell infiltration and antitumor activity [76]. As illustrated above in the preclinical examples, CAR T cells can be used to secrete factors that modify the TME, and potentially support a broader antitumor immune response. Numerous clinical trials using fourth-generation CAR T cells for the treatment of solid tumors are currently ongoing [58].

\section{Conclusion}

The importance of the cellular metabolism in regulating $\mathrm{T}$ cell function is becoming ever more evident. In order to mount an effective immune response $\mathrm{T}$ cells need adequate energy supplies. Unfortunately, tumor cells make use of similar metabolic pathways, namely they can upregulate glycolysis thereby depleting the tumor microenvironment of the glucose, a fuel vital to $\mathrm{T}$ cell function. At the same time, the tumor microenvironment can be severely hypoxic, leaving $\mathrm{T}$ cells without sufficient oxygen to use mitochondrial OXPHOS, while glucose levels can be insufficient for glycolysis. This leads to metabolic stress pushing $\mathrm{T}$ cells to exhaustion and tumor cells to grow unopposed.

This is especially relevant in solid tumors, as these are prone to containing hypoxic areas and hypoxia has a strong influence on cellular metabolic changes, regulated via the transcription factor HIF1- $\alpha$. Since hypoxic, highly glycolytic tumors are more aggressive it is pertinent that we gain a better understanding of $\mathrm{T}$ cell as well as tumor metabolic regulation; the former to increase the success of $T$ cell therapies especially in the context of targeting solid tumors and the latter to develop strategies of interfering with the tumor metabolism in order to slow down the tumor progression, make the tumor less aggressive and make the tumor a better target for the immune system.

\section{Future perspective}

In addition to the different mechanisms in the TME that suppress immune responses, the delivery of immune cells to tumors is literally a bottleneck in immunotherapy. After an initial avascular phase of tumor growth, and in order to sustain tumor proliferation, solid tumors recruit vessels by hypoxia-induced angiogenesis and formation of new vessels, but also by nonangiogenic mechanisms. The tumor vasculature structure and function are abnormal, resulting in hypoperfusion and hypoxia [77]. Furthermore, solid tumors are subjected to mechanical solid stress (forces present in and originating from the solid component of a tumor) [7]. Solid stress induces compression of blood vessels and worsening of hypoxia among other effects. Altogether, abnormal vasculature and solid stress hinders the transport of therapeutic cells and drugs. Strategies that improve vascular normalization, alleviate solid stress and decrease hypoxia should therefore enter the cancer immunotherapy toolbox. For example, the antihypertensive drug Losartan was shown to reduce solid stress and increase vascular perfusion [78], enhance the distribution of nanomedicine, oncolytic viruses [78,79] and drugs. Losartan thus represents an interesting example of a widely used drug, which may be crucial to enable the newest immunotherapies to fully exert their antitumor effect.

The increased knowledge about the inhibitory mechanisms in solid tumors combined with new ways of combating the immunosuppressive environment will enhance the likelihood of improved efficacy of future immunotherapeutic modalities. 
Executive summary

Metabolic regulation of T cells

- Upon activation T cells undergo metabolic alterations to increase glucose uptake and glycolysis for rapid production of energy and building blocks to support proliferation and effector functions.

- Inhibition of this metabolic switch (for example by limiting glucose) leads to T cell dysfunction and tumor infiltrating lymphocytes from low glucose tumors are functionally impaired.

- Even after glycolysis is increased, substrate and energy production by mitochondria play an important role in giving T cells full effector function. Exhausted T cells/tumor infiltrating lymphocytes (TIL) have mitochondrial deficiencies.

- New ways of generating therapeutic T cells with a metabolic profile able to withstand the nutrient deplete tumor environment are needed.

T cell exhaustion in the tumor microenvironment

- T cells encountering ongoing antigenic stimulation gradually become dysfunctional a state called exhaustion. Exhausted T cells highly express different combinations of multiple co-inhibitory molecules, the most prominent of which is Programmed Death-1.

- Accumulating data suggest that T cell exhaustion can also be driven by metabolic stress, such as low oxygen availability and nutrient deprivation, as encountered in the tumor microenvironment.

Suppressive mechanisms in the tumor microenvironment

- Tumor cells show marked alterations in their metabolic profiles allowing them to proliferate indefinitely. These cellular metabolic changes form part of the hallmarks of cancer and impact nutrient availability in the tumor microenvironment.

- Tumors are often hypoxic and this leads to increased utilization of glycolysis, depleting the tumor of glucose while lactate produced as waste product acidifies the tumor microenvironment. Lactate is directly immunosuppressive to T cells.

- There is an inverse correlation between the glycolytic activity of tumor cells and T cell function.

Hypoxia in tumors, impact of HIF- $1 \alpha$ on T cells

- Hypoxia induces the stabilization of the HIF-1 $\alpha$ transcription factor - a direct regulator of cellular metabolism leading to phenotypic and functional changes.

- Findings whether HIF-1 $\alpha$ stabilization in T cells enhances or inhibits T cell effector functions are controversial.

TILs \& chimeric antigen receptor T cells in solid tumors

- Adoptive cell therapy for the treatment of solid tumors can rely either on the infusion of polyclonal TILs, or of genetically engineered $T$ cells that express receptors ( $T$ cell receptor or chimeric antigen receptor [CAR]) specifically directed against tumor antigens.

- Solid tumors have their own microenvironment and this poses specific challenges for CAR T cells compared with hematological malignancies.

- Combination therapies, such as using check-point inhibitors and T cell therapy, may allow to increase efficacy of CAR T cell therapy.

Modulation of tumor environment by CAR T cells

- Fourth-generation CAR T cells, which have been modified to incorporate effector functions such as the production of inflammatory cytokines not naturally produced by T cells, may be able to overcome immunosuppression in the tumor microenvironment.

Financial \& competing interests disclosure

This study was supported by grants from Cancerfonden (4-251/2017), Dr Åke Olsson Foundation, Radiumhemmet, and the Mix private donation. The authors have no other relevant affiliations or financial involvement with any organization or entity with a financial interest in or financial conflict with the subject matter or materials discussed in the manuscript apart from those disclosed.

No writing assistance was utilized in the production of this manuscript.

\section{Open access}

This work is licensed under the Attribution-NonCommercial-NoDerivatives 4.0 Unported License. To view a copy of this license, visit http://creativecommons.org/licenses/by-nc-nd/4.0/ 


\section{References}

Papers of special note have been highlighted as: $\bullet$ of interest; $\bullet \bullet$ of considerable interest

1. Ledford H, Else H, Warren M. Cancer immunologists scoop medicine Nobel prize. Nature 562(7725), 20-21 (2018).

2. Eurostat, European Commission. https://ec.europa.eu/eurostat/web/products-eurostat-news/-/DDN-20181122-1?inheritRedirect=true

3. Anari F, Ramamurthy C, Zibelman M. Impact of tumor microenvironment composition on therapeutic responses and clinical outcomes in cancer. Future Oncol. 14(14), 1409-1421 (2018).

4. Buck MD, Sowell RT, Kaech SM, Pearce EL. Metabolic instruction of immunity. Cell 169(4), 570-586 (2017).

- Detailed review on metabolic regulation of immune cells, with a focus on $T$ cells.

5. Kosti P, Maher J, Arnold JN. Perspectives on chimeric antigen receptor T-cell immunotherapy for solid tumors. Front. Immunol. 9, 1104 (2018).

6. Zhang Y, Ertl HCJ. Starved and asphyxiated: how can CD8(+) T cells within a tumor microenvironment prevent tumor progression. Front. Immunol. 7, 32 (2016).

- The authors raise and discuss the idea of metabolic exhaustion.

7. Stylianopoulos T, Munn LL, Jain RK. Reengineering the physical microenvironment of tumors to improve drug delivery and efficacy: from mathematical modeling to bench to bedside. Trends Cancer 4(4), 292-319 (2018).

8. Vuillefroy De Silly R, Dietrich PY, Walker PR. Hypoxia and antitumor CD8(+) T cells: an incompatible alliance? Oncoimmunology 5(12), e1232236 (2016).

9. Le Q-T, Chen E, Salim A et al. An evaluation of tumor oxygenation and gene expression in patients with early stage non-small cell lung cancers. Clin. Cancer Res. 12(5), 1507-1514 (2006).

10. Graffman S, Björk P, Ederoth P, Ihse I. Polarographic pO2 measurements of intra-abdominal adenocarcinoma in connection with intraoperative radiotherapy before and after change of oxygen concentration of anaesthetic gases. Acta Oncol. 40(1), 105-107 (2001).

11. Scharping N, Delgoffe G. Tumor microenvironment metabolism: a new checkpoint for anti-tumor immunity. Vaccines 4(4), 46-15 (2016).

-. Elegant study showing how engineering the cellular metabolism can make $\mathrm{T}$ cells more effective in controlling solid tumors.

12. Maciver NJ, Michalek RD, Rathmell JC. Metabolic regulation of T lymphocytes. Annu. Rev. Immunol. 31(1), 259-283 (2013).

13. Warburg O. Respiratory impairment in cancer cells. Science 124(3215), 269-270 (1956).

14. Ho P-C, Bihuniak JD, Macintyre AN et al. Phosphoenolpyruvate is a metabolic checkpoint of anti-tumor T cell responses. Cell 162(6), 1217-1228 (2015).

15. Van Der Windt GJW, Everts B, Chang C-H et al. Mitochondrial respiratory capacity is a critical regulator of CD8+ T cell memory development. Immunity 36(1), 68-78 (2012).

16. Pallett LJ, Davies J, Colbeck EJ et al. IL-2(high) tissue-resident T cells in the human liver: sentinels for hepatotropic infection. J. Exp. Med. 214(6), 1567-1580 (2017).

17. Henson SM, Lanna A, Riddell NE et al. p38 signaling inhibits mTORC1-independent autophagy in senescent human CD8+ T cells. J. Clin. Invest. 124(9), 4004-4016 (2014).

18. Cao Y, Rathmell JC, Macintyre AN. Metabolic reprogramming towards aerobic Glycolysis correlates with greater proliferative ability and resistance to metabolic inhibition in CD8 versus CD4 T cells. PLoS ONE 9(8), e104104 (2014).

19. Bengsch B, Johnson AL, Kurachi M et al. Bioenergetic insufficiencies due to metabolic alterations regulated by the inhibitory receptor PD-1 are an early driver of CD8+ T cell exhaustion. Immunity 45(2), 358-373 (2016).

20. Schurich A, Pallett LJ, Jajbhay D et al. Distinct metabolic requirements of exhausted and functional virus-specific CD8 T cells in the same host. Cell Rep. 16(5), 1243-1252 (2016).

21. Kawalekar OU, O'connor RS, Fraietta JA et al. Distinct signaling of coreceptors regulates specific metabolism pathways and impacts memory development in CAR T cells. Immunity 44(2), 380-390 (2016).

22. Wherry EJ, Kurachi M. Molecular and cellular insights into T cell exhaustion. Nat. Rev. Immunol. 15(8), 486-499 (2015).

23. Wherry EJ, Blattman JN, Murali-Krishna K, Van Der Most R, Ahmed R. Viral persistence alters CD8 T-cell immunodominance and tissue distribution and results in distinct stages of functional impairment. J. Virol. 77(8), 4911-4927 (2003).

24. Wherry EJ, Ahmed R. Memory CD8 T-cell differentiation during viral infection. J. Virol. 78(11), 5535-5545 (2004).

25. Parry RV, Chemnitz JM, Frauwirth KA et al. CTLA-4 and PD-1 receptors inhibit T-cell activation by distinct mechanisms. Mol. Cell. Biol. 25(21), 9543-9553 (2005).

26. Frauwirth KA, Riley JL, Harris MH et al. The CD28 signaling pathway regulates glucose metabolism. Immunity 16(6), 769-777 (2002).

27. Hanahan D, Weinberg RA. Hallmarks of cancer: the next generation. Cell 144(5), 646-674 (2011).

28. Pucino V, Cucchi D, Mauro C. Lactate transporters as therapeutic targets in cancer and inflammatory disease. Expert Opin. Ther. Targets 22(9), 735-743 (2018). 
29. Brand A, Singer K, Koehl GE et al. LDHA-associated lactic acid production blunts tumor immunosurveillance by $\mathrm{T}$ and NK cells. Cell Metabolism 24(5), 657-671 (2016).

30. Dietl K, Renner K, Dettmer K et al. Lactic acid and acidification inhibit TNF secretion and glycolysis of human monocytes. J. Immunol. 184(3), 1200-1209 (2010).

31. Cascone T, Mckenzie JA, Mbofung RM et al. Increased Tumor Glycolysis Characterizes Immune Resistance to Adoptive T Cell Therapy. Cell Metab. 27(5), 977-987 (2018).

32. Feng J, Yang H, Zhang Y et al. Tumor cell-derived lactate induces TAZ-dependent upregulation of PD-L1 through GPR81 in human lung cancer cells. Oncogene 36(42), 5829-5839 (2017).

33. Doedens AL, Phan AT, Stradner MH et al. Hypoxia-inducible factors enhance the effector responses of CD8(+) T cells to persistent antigen. Nat. Immunol. 14(11), 1173-1182 (2013).

34. Gacerez AT, Sentman CL. T-bet promotes potent antitumor activity of CD4+ CAR T cells. Cancer Gene Ther. 25(5-6), 117-128 (2018).

35. Gillies RJ, Gatenby RA. Metabolism and its sequelae in cancer evolution and therapy. Cancer J. 21(2), 88-96 (2015).

36. Chang C-H, Qiu J, O'sullivan D et al. Metabolic competition in the tumor microenvironment is a driver of cancer progression. Cell 162(6) 1229-41 (2015).

37. Hsu TS, Lai MZ. Hypoxia-inducible factor 1alpha plays a predominantly negative role in regulatory T cell functions. J. Leukoc. Biol. 104(5), 911-918 (2018).

38. Vaupel P, Mayer A. Hypoxia in cancer: significance and impact on clinical outcome. Cancer Metastasis Rev. 26(2), 225-239 (2007).

39. Gray LH, Conger AD, Ebert M, Hornsey S, Scott OC. The concentration of oxygen dissolved in tissues at the time of irradiation as a factor in radiotherapy. Br. J. Radiol. 26(312), 638-648 (1953).

40. Brahimi-Horn MC, Pouyssegur J. HIF at a glance. J. Cell. Sci. 122(Pt 8), 1055-1057 (2009).

41. Corcoran SE, O'neill LA. HIF1alpha and metabolic reprogramming in inflammation. J. Clin. Invest. 126(10), 3699-3707 (2016).

42. Talks KL, Turley H, Gatter KC et al. The expression and distribution of the hypoxia-inducible factors HIF-1alpha and HIF-2alpha in normal human tissues, cancers, and tumor-associated macrophages. Am. J. Pathol. 157(2), 411-421 (2000).

43. Palazon A, Goldrath AW, Nizet V, Johnson RS. HIF transcription factors, inflammation, and immunity. Immunity 41(4), 518-528 (2014).

44. Shi LZ, Wang R, Huang G et al. HIF1alpha-dependent glycolytic pathway orchestrates a metabolic checkpoint for the differentiation of TH17 and Treg cells. J. Exp. Med. 208(7), 1367-1376 (2011).

45. Dang EV, Barbi J, Yang HY et al. Control of T(H)17/T(reg) balance by hypoxia-inducible factor 1. Cell 146(5), 772-784 (2011).

46. Feldhoff LM, Rueda CM, Moreno-Fernandez ME et al. IL-1beta induced HIF-1alpha inhibits the differentiation of human FOXP3(+) T cells. Sci. Rep. 7(1), 465 (2017).

47. Clambey ET, Mcnamee EN, Westrich JA et al. Hypoxia-inducible factor-1 alpha-dependent induction of FoxP3 drives regulatory T-cell abundance and function during inflammatory hypoxia of the mucosa. Proc. Natl Acad. Sci. USA 109(41), E2784-2793 (2012).

48. Ben-Shoshan J, Maysel-Auslender S, Mor A, Keren G, George J. Hypoxia controls CD4+CD25+ regulatory T-cell homeostasis via hypoxia-inducible factor-1alpha. Eur. J. Immunol. 38(9), 2412-2418 (2008).

49. Thiel M, Caldwell CC, Kreth $\mathrm{S}$ et al. Targeted deletion of HIF-1alpha gene in T cells prevents their inhibition in hypoxic inflamed tissues and improves septic mice survival. PLoS ONE 2(9), e853 (2007).

50. Zhang Y, Kurupati R, Liu L et al. Enhancing CD8(+) T cell fatty acid catabolism within a metabolically challenging tumor microenvironment increases the efficacy of melanoma immunotherapy. Cancer Cell 32(3), 377-391 e379 (2017).

51. Palazon A, Tyrakis PA, Macias D et al. An HIF-1alpha/VEGF-A axis in cytotoxic T cells regulates tumor progression. Cancer Cell 32(5), 669-683 e665 (2017).

52. Xu Y, Chaudhury A, Zhang M et al. Glycolysis determines dichotomous regulation of T cell subsets in hypoxia. J. Clin. Invest. 126(7), 2678-2688 (2016).

53. Duhen T, Duhen R, Montler R et al. Co-expression of CD39 and CD103 identifies tumor-reactive CD8 T cells in human solid tumors. Nat. Commun. 9(1), 2724 (2018).

54. Simoni $\mathrm{Y}$, Becht E, Fehlings $\mathrm{M}$ et al. Bystander CD8(+) T cells are abundant and phenotypically distinct in human tumour infiltrates. Nature 557(7706), 575-579 (2018).

55. Rosenberg SA, Packard BS, Aebersold PM et al. Use of tumor-infiltrating lymphocytes and interleukin-2 in the immunotherapy of patients with metastatic melanoma. A preliminary report. N. Engl. J. Med. 319(25), 1676-1680 (1988).

-. First report of the ability of tumor-infiltrating lymphocytes-based adoptive cell transfer to mediate cancer regression in patients with metastatic melanoma.

56. Fournier C, Martin F, Zitvogel L, Kroemer G, Galluzzi L, Apetoh L. Trial watch: adoptively transferred cells for anticancer immunotherapy. Oncoimmunology 6(11), e1363139 (2017). 
57. Rosenberg SA, Yang JC, Sherry RM et al. Durable complete responses in heavily pretreated patients with metastatic melanoma using T-cell transfer immunotherapy. Clin. Cancer Res. 17(13), 4550-4557 (2011).

58. Knochelmann HM, Smith AS, Dwyer CJ, Wyatt MM, Mehrotra S, Paulos CM. CAR T cells in solid tumors: blueprints for building effective therapies. Front. Immunol. 9, 948501-948520 (2018).

59. June CH, Sadelain M. Chimeric antigen receptor therapy. N. Engl. J. Med. 379(1), 64-73 (2018).

60. Chen N, Li X, Chintala NK, Tano ZE, Adusumilli PS. Driving CARs on the uneven road of antigen heterogeneity in solid tumors. Curr. Opin. Immunol. 51, 103-110 (2018).

61. Srivastava S, Riddell SR. Chimeric antigen receptor T cell therapy: challenges to bench-to-bedside efficacy. J. Immunol. 200(2), 459-468 (2018).

62. Morgan RA, Yang JC, Kitano M, Dudley ME, Laurencot CM, Rosenberg SA. Case report of a serious adverse event following the administration of T cells transduced with a chimeric antigen receptor recognizing ERBB2. Mol. Ther. 18(4), 843-851 (2010).

63. Chong EA, Melenhorst JJ, Lacey SF et al. PD-1 blockade modulates chimeric antigen receptor (CAR)-modified T cells: refueling the CAR. Blood 129(8), 1039-1041 (2017).

64. Heczey A, Louis CU, Savoldo B et al. CAR T Cells Administered in Combination with Lymphodepletion and PD-1 Inhibition to patients with neuroblastoma. Mol. Ther. 25(9), 2214-2224 (2017).

65. Curtsinger JM, Mescher MF. Inflammatory cytokines as a third signal for T cell activation. Curr. Opin. Immunol. 22(3), 333-340 (2010).

66. Sadelain M, Brentjens R, Rivière I. The basic principles of chimeric antigen receptor design. Cancer Discov. 3(4), 388-398 (2013).

67. Jaspers JE, Brentjens RJ. Development of CAR T cells designed to improve antitumor efficacy and safety. Pharmacol. Ther. 178, 83-91 (2017).

68. Jarnicki AG, Lysaght J, Todryk S, Mills KHG. Suppression of antitumor immunity by IL-10 and TGF-beta-producing T cells infiltrating the growing tumor: influence of tumor environment on the induction of CD4+ and CD8+ regulatory T cells. J. Immunol. 177(2), 896-904 (2006).

69. Bajgain P, Tawinwung S, D'elia L et al. CAR T cell therapy for breast cancer: harnessing the tumor milieu to drive $\mathrm{T}$ cell activation. $J$. Immunother. Cancer 6(1), 34 (2018).

70. Sukumaran S, Watanabe N, Bajgain P et al. Enhancing the potency and specificity of engineered T cells for cancer treatment. Cancer Discov. 8(8), 972-987 (2018).

71. Pegram HJ, Lee JC, Hayman EG et al. Tumor-targeted T cells modified to secrete IL-12 eradicate systemic tumors without need for prior conditioning. Blood 119(18), 4133-4141 (2012).

- Demonstrates that IL-12-secreting CD19-CAR T cells were able to overcome inhibition of CAR T-cell activity by regulatory T cells and eliminated the need for prior conditioning chemotherapy.

72. Hu B, Ren J, Luo Y et al. Augmentation of antitumor immunity by human and mouse CAR T cells secreting IL-18. Cell Rep. 20(13), 3025-3033 (2017).

73. Adachi K, Kano Y, Nagai T, Okuyama N, Sakoda Y, Tamada K. IL-7 and CCL19 expression in CAR-T cells improves immune cell infiltration and CAR-T cell survival in the tumor. Nat. Biotechnol. 36(4), 346-351 (2018).

74. Suarez ER, Chang De K, Sun J et al. Chimeric antigen receptor T cells secreting anti-PD-L1 antibodies more effectively regress renal cell carcinoma in a humanized mouse model. Oncotarget 7(23), 34341-34355 (2016).

75. Ligtenberg MA, Mougiakakos D, Mukhopadhyay M et al. Coexpressed catalase protects chimeric antigen receptor-redirected T cells as well as bystander cells from oxidative stress-induced loss of antitumor activity. J. Immunol. 196(2), 759-766 (2016).

76. Caruana I, Savoldo B, Hoyos V et al. Heparanase promotes tumor infiltration and antitumor activity of CAR-redirected T lymphocytes. Nat. Med. 21(5), 524-529 (2015).

77. Schaaf MB, Garg AD, Agostinis P. Defining the role of the tumor vasculature in antitumor immunity and immunotherapy. Cell Death Dis. 9(2), 115 (2018).

78. Pinter M, Jain RK. Targeting the renin-angiotensin system to improve cancer treatment: implications for immunotherapy. Sci. Transl. Med. 9(410), eaan5616 (2017).

79. Guo ZS, Liu Z, Kowalsky S et al. Oncolytic immunotherapy: conceptual evolution, current strategies, and future perspectives. Front. Immunol. 8, 1686 (2017). 